\title{
Mechanical and thermal properties of sugar palm fiber (Arenga pinnata) - reinforced phenolic composites
}

\begin{abstract}
The aim of Chapter 7 is to investigate the mechanical and thermal performance of sugar palm fibers (SPF) reinforced with phenolic composites. SPF in powdered form (i.e., $\leq 150 \mu \mathrm{m}$ ) with five different fiber loadings ranging in volume from $0 \%$ to $40 \%$ was used to fabricate the composite samples with a hot press. Flexural, impact, and compressive properties of the composites were analyzed, while the thermal behavior was analyzed by means of thermogravimetric analysis. Scanning electron microscopy was used to examine the morphology of the fracture surface of the samples after impact testing. The results showed that an increase in the filler loadings of the SPFs in the phenolic composites improved the mechanical properties of the composites up to $30 \mathrm{vol} \%$. Scanning electron microscopy images of the 30 vol\% SPF composite displayed good fiber dispersion. Thermal degradation showed that the addition of SPF reduced the thermal stability of the composites. Consequently, SPF can be effectively used as an alternative natural fiber for reinforcing biocomposites, particularly in tribology applications.
\end{abstract}

Keywords: Particle-reinforced composites; Polymers; Mechanical properties; Thermogravimetric analysis 